19 Revue d'histoire du XIXe siècle

Société d'histoire de la révolution de 1848 et des

révolutions du XIXe siècle

47 | 2013

Quel est l'avenir du XIX $\mathrm{X}^{\mathrm{e}}$ siècle ?

\title{
La bibliothèque de Maurice Agulhon : cadre de travail, coulisses de l'écriture, et patrimoine scientifique
}

Jonathan Barbier et Natalie Petiteau

\section{OpenEdition}

Journals

Édition électronique

URL : http://journals.openedition.org/rh19/4565

DOl : $10.4000 /$ rh 19.4565

ISSN : 1777-5329

Éditeur

La Société de 1848

Édition imprimée

Date de publication : 31 décembre 2013

Pagination : $95-112$

ISSN : 1265-1354

\section{Référence électronique}

Jonathan Barbier et Natalie Petiteau, « La bibliothèque de Maurice Agulhon : cadre de travail, coulisses de l'écriture, et patrimoine scientifique », Revue d'histoire du XIXe siècle [En ligne], 47 | 2013, mis en ligne le 31 décembre 2016, consulté le 19 avril 2019. URL : http://journals.openedition.org/rh19/4565 ; DOI $10.4000 /$ rh 19.4565 


\section{JONATHAN BARBIER, NATALIE PETITEAU}

\section{La bibliothèque de Maurice Agulhon: cadre de travail, coulisses de l'écriture, et patrimoine scientifique}

Dans l'histoire des bibliothèques qui est en cours d'élaboration, on a encore peu eu l'occasion de s'intéresser aux bibliothèques privées. L'historien Maurice Agulhon nous permet de le faire grâce au soin qu'il a pris du devenir de sa bibliothèque de travail. Dès 2007 en effet, il a souhaité donner à l'université d'Avignon une partie de ses livres, ceux dont il estimait qu'ils étaient utiles à des étudiants et dont il jugeait possible de se séparer en raison du fait qu'il n'en avait plus l'usage. Un inventaire a été dressé à l'issue des deux vagues successives de dons à l'universitée. Parallèlement, l'université de Lyon II acceptait de réunir au fonds d'Avignon ce que Maurice Agulhon avait donné à la suite de l'incendie de la bibliothèque universitaire de Lyon et qui n'avait finalement pas encore été référencé dans le laboratoire où ils étaient déposés.

Mais le cœur de la bibliothèque de Maurice Agulhon demeure dans sa maison familiale de Villeneuve-lez-Avignon. Celle-ci abrite bien évidemment les œuvres littéraires dont il s'est nourri, et qui n'ont pour l'heure fait l'objet d'aucun inventaire : mais on trouve sur les rayons de la bibliothèque de sa chambre tous les classiques de la littérature, y compris une édition originale reliée cuir de l'œuvre de Zola. S'y trouvent aussi, bien évidemment, Balzac, Victor Hugo, Flaubert, Lamartine, Baudelaire, Eugène Sue, Vallès, Renan, Erckmann et Chatrian, Daudet, Loti, mais encore les Goncourt, Flora Tristan et Martin Nadaud, etc.

De ses livres scientifiques, rangés dans son bureau, Maurice Agulhon a élaboré un classement systématique, signalé par des étiquettes apposées sur chaque rayonnage. Ce classement est une construction progressive, il a été voulu par Maurice Agulhon quand il s'est préoccupé du devenir de sa

1. Nous remercions très vivement le professeur Maurice Agulhon pour son aide et son accueil. 2. L'inventaire du fonds Agulhon de la bibliothèque de l'université d'Avignon est disponible également en ligne et peut être interrogé spécifiquement par le biais de la rubrique "fonds spécialisés" : http://www.bu.univ-avignon.fr/cda/. Nous remercions très vivement l'université d'Avignon et ses personnels pour leur aide, et particulièrement Madame Isabelle Dimondo, conservatrice de la bibliothèque universitaire, pour son soutien constant et sans faille. 
bibliothèque : à partir du moment où il a songé à ouvrir celle-ci aux chercheurs, il a commencé à réfléchir à la façon de l'organiser. Au fil des rencontres avec les auteurs du présent article, ce classement s'est affiné, précisé, modifié. Il est donc une relecture a posteriori par laquelle Maurice Agulhon a eu le souci de faire apparaître clairement la culture dont son œuvre est issue. Il a également souhaité mettre en évidence la façon dont sa bibliothèque reflète les thèmes qui ont été au cœur de ses recherches. Il s'est heurté bien évidemment à la difficulté de fixer une catégorie pour certains ouvrages qui peuvent s'inscrire dans plusieurs thématiques. Certains livres peuvent changer de place au gré des réflexions nouvelles de Maurice Agulhon. Soucieux de la cohérence scientifique de sa bibliothèque, il est également très préoccupé de son devenir matériel. Il souhaite la constituer en un patrimoine utile à la communauté scientifique, car elle rend compte de son travail d'historien.

Mais étant dans l'impossibilité de résoudre la question de ce qu'il adviendra de cette bibliothèque après sa mort, il a accepté une sauvegarde virtuelle de celle-ci. L'université d'Avignon a donc financé l'inventaire des livres de Villeneuve afin de connaître la totalité de ce qui a constitué cet imposant instrument de travail, inventaire aujourd'hui disponible sur le site de la bibliothèque universitaire d'Avignon ${ }^{3}$. Ce faisant, les dédicaces les plus importantes ont été photographiées et seront ultérieurement mises en ligne. Enfin, toujours avec le soutien financier et matériel de l'université d'Avignon, a été réalisé un film qui permet une visite virtuelle de la bibliothèque, y compris avec les objets symboliques qui en font l'ornementation : la décision a été prise de procéder à ce complément de la sauvegarde virtuelle de la bibliothèque quand il est apparu qu'il sera sans doute impossible de rendre ces lieux facilement accessibles aux chercheurs ${ }^{4}$. Ainsi, dans l'hypothèse où les historiens ne pourront pas travailler dans cette bibliothèque, ils pourront néanmoins savoir exactement de quoi elle était composée et comment elle était organisée.

De telles bibliothèques sont aujourd'hui en voie de disparition : les nouvelles générations d'historiens ont bien plus l'habitude des consultations en ligne et des lectures d'ouvrages électroniques. La bibliothèque de Maurice Agulhon ainsi filmée et inventoriée est un rare témoignage de ce type d'instrument de travail constituant en même temps un cadre de réflexion et d'écriture. Il n'est pas innocent que Maurice Agulhon ait aimé agrémenter ses murs ou ses étagères de représentations de Marianne ou d'objets témoignant des cadeaux faits par de lointains étudiants chinois ou américains.

3. http://www.bu.univ-avignon.fr/skins/EXPLOITATION/images/Portal/inventaire\%20agulhon. pdf : afin d'alléger ce texte, on ne donnera pas en note de bas de page, sauf pour éviter toute confusion éventuelle, les références des ouvrages mentionnés, qui figurent toutes dans cet inventaire auquel le lecteur peut aisément se reporter.

4. http://www.bu.univ-avignon.fr/skins/EXPLOITATION/images/Portal/docu_agulhon.mov. 
La bibliothèque permet par elle-même de prendre la mesure de l'apport de son propriétaire, internationalement reconnu pour avoir renouvelé en profondeur la compréhension de la vie politique des temps contemporains et montré la pertinence des réflexions sur des objets nouveaux, sociabilité et symboles notamment. C'est pourquoi on tentera ici de montrer ce que la composition de ce fonds peut apporter à la compréhension de l'œuvre de l'historien, ce que les dédicaces aussi - source jamais encore exploitée jusqu'ici - révèlent de son rayonnement. La bibliothèque de Maurice Agulhon, comme toute bibliothèque de particulier, est le fruit d'une lente et complexe constitution, au gré des achats, des dons de collègues et amis, des envois d'éditeurs. Comme toute bibliothèque de particulier, l'accumulation des ouvrages y est d'une part le fruit de certains hasards - ceux des envies ponctuelles de lecture, comme ceux des envois de certains auteurs -, d'autre part d'une démarche systématique dans certaines directions de recherche - ce qui suscite à la fois achats et envois de la part de collègues et de disciples. Son analyse s'annonce de ce fait périlleuse : «lorsqu'on est en face d'un fonds particulier, la question à se poser est celle de sa logique et de sa cohérence. [...] Il convient d'envisager un fonds particulier comme un système cohérent obéissant à une logique propre $"^{5}$.

Cette bibliothèque met en évidence le rapport entre une tradition, une culture et l'évolution d'une ouvre d'historien. Elle reflète plusieurs facettes de Maurice Agulhon : son histoire personnelle, son travail d'historien, son rôle de commissaire d'exposition. Le fonds est majoritairement consacré à la discipline historique, même s'il est pluri-disciplinaire (histoire, géographie, sociologie, sciences politiques, histoire de l'art...). Il donne à lire son itinéraire intellectuel.

*

\section{UN FONDS PATRIMONIAL}

POUR LA RECHERCHE EN SCIENCES HUMAINES

\section{Une bibliothèque riche de trois mille ouvrages}

On peut diviser la bibliothèque de Maurice Agulhon en deux parties : il existe un fonds ancien, relevant de l'héritage familial, et un fonds moderne, résultant d'achats et de dons. Le fonds ancien est la partie la plus précieuse de la bibliothèque en raison de l'ancienneté des ouvrages, mais aussi des informations qu'il livre sur l'éducation et les disciplines préférées du jeune Maurice Agulhon. Car il a pris soin de conserver les livres de sa famille et de son enfance. Certains ouvrages reflètent le cadre culturel dans lequel ses

5. Cécile Rothlin, Appréhender un fonds particulier : le fonds Ben Elek à la Bibliothèque interuniversitaire Lettres et sciences humaines à Lyon, Mémoire d'étude pour le diplôme de conservateur de bibliothèque, sous la direction de Dominique Varry, Villeurbanne, ENSSIB, 2005, p. 25-26 
parents ont grandi, tel ce livre de Chants populaires pour les écoles, datant de 1913 et ayant appartenu à sa mère. Les textes de ces chants, au caractère patriotique et guerrier, contrastent avec le rejet de tout bellicisme qui a distingué les parents de Maurice Agulhon. Le fonds ancien est également composé des livres offerts au lycéen, tel ce livre pédagogique sur la science et vie de la terre reçu pour l'anniversaire de ses treize ans $s^{6}$. Certains titres, comme Le Petit fauconnier de Louis XIII de Jules Chancel, ou Le conscrit de 1813 d'Emile Erckmann et Alexandre Chatrian, dessinent les voies suivant lesquelles Maurice Agulhon a pu trouver le goût de l'histoire. Les autres ouvrages anciens sont, pour la plupart, des récompenses reçues dans un cadre scolaire, témoignant des matières dans lesquelles il excellait au lycée Frédéric Mistral d'Avignon, qu'il a fréquenté de 1936 à 1943 : il décroche trois prix $\mathrm{d}^{\prime}$ excellence en classe de sixième, cinquième et seconde ${ }^{7}$.

Le fonds moderne est, quant à lui, le produit des achats ou des échanges avec les amis et les pairs. Il mérite tout autant l'attention des chercheurs ${ }^{8}$ car il est le miroir des différents champs de recherche de Maurice Agulhon : la sociabilité et l'histoire du folklore, l'histoire politique et l'histoire de la Deuxième République, l'histoire symbolique à travers la figure de Marianne ou un nouveau regard porté sur Charles de Gaulle. Cependant, ce fonds moderne permet aussi de constater un travail pluridisciplinaire. L'esprit de l'historien s'est ouvert aux méthodes et aux grilles de lecture des autres sciences humaines : la sociologie et l'anthropologie, les sciences politiques, l'histoire de l'art et les "théories sur l'imagerie». On peut observer également un intérêt de l'historien "quarante-huitard" pour des champs historiques qu'il n'a traités que partiellement. Ce n'est pas parce que Maurice Agulhon n'a pas écrit, par exemple, de livre sur l'histoire des femmes ou l'histoire du corps qu'il ne s'y est pas intéressé.

\section{Les logiques d'un classement: les sections thématiques}

La cohérence de la bibliothèque est manifeste dans le regroupement des livres autour de grands thèmes: l'héritage et les initiations, l'histoire de France de la Révolution française à nos jours, l'histoire contemporaine par thèmes, les rencontres diverses, l'histoire régionale, l'historiographie. Sont également mis en exergue les derniers objets d'étude de Maurice Agulhon, c'est-à-dire Marianne et Charles De Gaulle. Viennent enfin des sections

6. Henri Collin Delavaud, Vers l'inconnu : les hommes à la découverte de la terre, Paris, Bourrelier, 1939. 7. James Fenimore Cooper, Le Dernier des Mohicans, Liège, Gordinne, 1936; Percival Christopher Wren, Beau geste, Paris, Larousse, 1931; informations communiquées par Maurice Agulhon.

8. «Une vision du patrimoine qui s'articule de manière préférentielle, voire exclusive, sur les fonds anciens conduit à sous-estimer l'intérêt patrimonial à venir des autres collections, notamment contemporaines, alors qu'il est indispensable d'admettre que leur transmission, conjointement aux traces que nous ont laissées les époques antérieures, présente le même degré de nécessité» : Bernard Huchet, "Patrimoine et politique documentaire : la bibliothèque dans son entier» in Dominique Coq [dir.], Apprendre à gérer des collections patrimoniales en bibliothèque, Villeurbanne, Presses de l'ENSSIB, 2012, p. 29-30. 
consacrées d'une part à sa propre production, d'autre part à celle de ses proches : Maurice Agulhon a retenu la formule «moi et les miens" pour rassembler les livres de ses amis et de ses adversaires (on y trouve donc Marc Fumaroli), de ses disciples les plus proches, des personnalités historiennes et non-historiennes qui ont eu une influence réelle dans ses recherches historiques. Un même thème peut, néanmoins, contenir diverses acceptions. Ainsi celui de l'héritage met en évidence les influences familiales, mais le cadre politique y a aussi sa place, ainsi que les écrits concernant le communisme et le socialisme. L'héritage régional renvoie aux origines méridionales tout autant qu'aux premiers terrains d'études. Le thème des rencontres a lui aussi plusieurs significations. Il évoque les champs d'études historiques sur lesquels Maurice Agulhon a pénétré au fil de sa formation, de ses cours et de ses recherches : l'histoire des nations étrangères, l'histoire mondiale et européenne, les arts, les religions. Mais les rencontres sont aussi celles qui ont été faites au fur et à mesure de l'entrée dans les divers réseaux d'historiens, d'universitaires, d'intellectuels, d'amis.

C'est dans les sections thématiques que Maurice Agulhon a placé les ouvrages portant sur les cercles et les chambrées, les sociabilités, la francmaçonnerie, les intellectuels et la laïcité. Une deuxième section est consacrée à l'histoire du genre et du corps, une troisième s'intéresse à l'analyse du $\mathrm{XIX}^{\mathrm{e}}$ siècle par le biais de l'histoire sociale. Les livres de Maurice Agulhon ont bien sûr leur propre section. On peut y consulter les titres qui ont fait la célébrité de l'auteur, de La République au village aux Marianne et à De Gaulle. On peut même observer que certains titres ont été traduits dans des langues étrangères, y compris en coréen, en chinois ou en grec. Cette section rassemble aussi soigneusement les ouvrages contenant des préfaces signées par Maurice Agulhon, ainsi que les livres collectifs contenant ses articles.

Ce fonds témoigne des préoccupations pluri-disciplinaires de son propriétaire. Citons les sections «sociologues et politistes» et «textes non historiens». Maurice Agulhon s'est imprégné du travail des anthropologues pour renouveler l'histoire sociale et l'histoire politique. Il est aussi un intellectuel passionné par les autres sciences humaines, et même par les sciences dures : on trouve ainsi des ouvrages de Denis Buican sur le darwinisme et l'évolutionnisme, les livres de Jean-Pierre Changeux sur la raison et la mémoire, les œuvres de vulgarisation d'Yves Coppens; les approches anthropologiques de l'antiquité grecque par Marcel Détienne; les travaux linguistiques de Claude Hagège; des essais du philosophe Michel Serres. Mais les caricatures de Plantu ou les albums d'Astérix ont été, à leur manière, une source d'inspiration pour certains articles de Maurice Agulhon' et ont aussi trouvé leur place dans cette bibliothèque qui couvre donc un large périmètre.

9. Maurice Agulhon, «Le mythe gaulois : Astérix. Un mythe et ses figures», Ethnologie française, volume $28, \mathrm{n}^{\circ} 3,1998$, p. 296-302. 


\section{Les leçons d'un inventaire : la chronologie couverte par la bibliothèque}

La chronologie que couvre cette bibliothèque s'étend du XVIII ${ }^{e}$ siècle jusqu'à aujourd'hui : hormis ceux provenant de ses pairs et amis, les ouvrages portant sur des périodes antérieures ont été pour la plupart légués à la bibliothèque universitaire d'Avignon, Maurice Agulhon ne les ayant pas considérés comme constitutifs de sa "bibliothèque de travail». Une des sections de la bibliothèque porte sur l'histoire de France "de la Révolution française jusqu'à la Commune». L'année 1848 occupe, de façon attendue, une place spécifique dans les temps forts mis en évidence. Une section lui est entièrement consacrée et est intitulée "Première approche d'historien : romantisme et 1848 ". Deux autres sections concernant l'histoire de la politique française ont fait l'objet d'un découpage chronologique plus fin : «Politique française : histoire républicaine 1871-1968", où la Commune est privilégiée, et "Politique française : crise actuelle». Maurice Agulhon s'interroge en ce début de XXI ${ }^{e}$ siècle sur les changements, les ruptures qui agitent le milieu politique via la question des mythes, de la personnification politique ou de la communication politique. Cette bibliothèque prend en compte les nouvelles problématiques du XXI siècle et Maurice Agulhon s'efforce d'enrichir ce fonds en suivant l'actualité des publications historiques et de sciences humaines : elle ne cesse pas, ainsi, de rendre compte de son parcours intellectuel. Mais elle témoigne aussi des va-et-vient de son propriétaire à travers l'histoire contemporaine.

\section{UN FONDS PARTICULIER, REFLET D'UN ITINÉRAIRE INTELLECTUEL}

\section{L'apprentissage de l'histoire et de la recherche}

S'il est une figure politique qui a marqué Maurice Agulhon, c'est celle de Jean Jaurès. Elle est pour lui à la fois un héritage familial, politique et historique. Il a écrit des articles sur ce théoricien socialiste ${ }^{10}$, et l'un de ses derniers travaux, qu'il co-signe avec Jean-François Chanet, consiste en une présentation de ses œuvres complètes. Les livres de Jaurès sont une composante essentielle du fonds Agulhon qui rassemble, de plus, une grande partie des biographies qui lui sont consacrées. Observons enfin la présence des publications de la Société d'Études Jaurésiennes et de la collection des Cahiers Jaurès.

Le communisme est, avec le socialisme de Jaurès, l'autre courant politique qui a influencé le citoyen et historien Maurice Agulhon. Celui-ci a adhéré aux idées communistes lorsqu'il était normalien. Même si, dès la fin

10. Maurice Agulhon, "Jaurès vu par Georges Renard et Louis Andrieux ", Bulletin de la Société d'études jaurésiennes, $\mathrm{n}^{\circ}$ 54, 1974, p. 13-15; "La fortune des Jaurès sous le Premier Empire ", Bulletin de la Société d'études jaurésiennes, $\mathrm{n}^{\circ}$ 56, 1975, p. 8; "Après les agressions contre les monuments Jaurès", Bulletin de la Société d'études jaurésiennes, $\mathrm{n}^{\circ} 84,1982$, p. 13-14; "La pensée de Jaurès et la défense nationale», Bulletin de la Société des études jaurésiennes, $\mathrm{n}^{\circ} 130$, Jaurès et la défense nationale, 1992, p. $49-62$. 
des années 1960, Maurice Agulhon s'est éloigné du Parti communiste, il ne renie pas, cependant, les apports du marxisme dans la recherche historique. Les travaux d'Ernest Labrousse et d'Albert Soboul, d'essence marxiste, ont conduit Maurice Agulhon, dans sa thèse, à faire le lien entre le social et le politique. Selon Maurice Agulhon, Karl Marx a conduit les historiens à regarder les événements du point de vue des classes populaires, des classes ouvrières, des anonymes et plus seulement du point de vue des institutions politiques et des grands hommes. On comprend donc que les œuvres de Karl Marx soient au cœur de sa bibliothèque originelle.

L'une de ses sections est en effet dédiée à la littérature marxiste et communiste. Elle a pour nom : «Expérience du communisme et ses suites». On y trouve des livres de penseurs marxistes et communistes, de Karl Marx à Maurice Thorez, en passant par Engels, Lénine, Staline, Paul Lafargue ou Charles Tillon. Maurice Agulhon souligne aujourd'hui que cette littérature peut être contestée, mais elle n'en est pas moins le témoignage d'une idéologie qui a marqué, pour le meilleur et pour le pire, les sociétés du XIX et $\mathrm{du} \mathrm{XX}^{\mathrm{e}}$ siècles. Les écrivains libertaires et marxistes, héritiers de l'auteur du Capital, ne sont pas absents : on lit ainsi les noms de Régis Debray, Louis Althusser ou encore de l'ancien Premier ministre socialiste, Lionel Jospin. On peut ajouter à cet ensemble sur la pensée communiste les manifestes, brochures, livres édités par le Parti communiste français. Mais cette section n'est pas uniquement représentative de la diversité des pensées communistes. Elle témoigne aussi du regard que les historiens et géographes ont porté sur les mouvements et partis communistes: on peut consulter des ouvrages du géographe Pierre George et ceux d'historiens comme Jean-Jacques Becker, Marc Ferro, François Furet, Annie Kriegel, Danielle Tartakowsky. Tous ces travaux scientifiques montrent à la fois l'implication d'un grand nombre de ces intellectuels dans l'aventure communiste, leurs analyses critiques vis-vis de l'histoire du parti et leur prise de recul face à leurs propres engagements.

Quant aux apprentissages spécifiquement historiens de Maurice Agulhon, ils se sont faits dans la khâgne de Lyon puis à l'École normale supérieure de la rue d'Ulm, mais aussi dans la lecture des publications qui marquaient alors les étudiants, de la collection "Clio » à celle de «L'évolution de l'humanité» ou de «Peuples et civilisations». Mais Maurice Agulhon, lorsqu'il a souhaité faire du tri dans sa bibliothèque, a confié à la bibliothèque universitaire d'Avignon bon nombre de ses ouvrages d'étudiant qui ne lui étaient plus d'aucune utilité. C'est donc de l'autre côté du Rhône qu'on peut aujourd'hui les trouver : il a lu dans Henri Sée Les origines du capitalisme moderne (1946) et L'histoire économique de la France (1948), il a découvert avec Philippe Sagnac La formation de la société française moderne (1946), il s'est nourri de l'œuvre de Georges Lefebvre tant à propos de La Révolution française (1951) que de Napoléon (1935), l'ouvrage de Louis Villat lui ayant également permis d'apprendre l'histoire de La Révolution et l'Empire (1947). D'Ernest Labrousse, il possédait Le mouvement ouvrier et 
les idées sociales en France de 1815 à la fin du XIXe siècle (1948), il s'est initié à l'Histoire religieuse de la France contemporaine (1948) dans Adrien Dansette, il a appris La formation de la population parisienne au XIXe siècle grâce à Louis Chevalier (1949), il a découvert les mécanismes de La monarchie parlementaire avec Félix Ponteil (1949) et Les institutions de la France avec Jacques Godechot (1951). Mais l'œuvre d'Albert Soboul demeure présente dans la bibliothèque de Villeneuve. Celle-ci est également pourvue d'ouvrages sur la sociabilité ou le compagnonnage durant le premier XIX ${ }^{\mathrm{e}}$ siècle. À ne prendre en considération que les livres que Maurice Agulhon a souhaité conserver chez lui, on constate que ce sont les œuvres qui concernent le communisme qui forment la majorité de la bibliothèque antérieure aux années 1960. Mais en analysant l'ensemble de sa bibliothèque, on découvre une collection qui forme un fonds très complet d'historien s'intéressant au XIX ${ }^{\mathrm{e}}$ siècle.

Riche de cette formation, Maurice Agulhon, par affiliation labroussienne, a donc orienté ses recherches vers l'histoire politique et sociale du premier $\mathrm{XIX}^{\mathrm{e}}$ siècle. On sait comment Ernest Labrousse attribuait à ses doctorants des départements choisis au gré des affectations dans le secondaire. C'est donc le Var qui échoit à Maurice Agulhon, nommé en tant que nouvel agrégé au lycée de Toulon. Mais, à son grand regret, un Var du premier XIX ${ }^{e}$ siècle, puisque l'après 1851 , qu'il convoitait, était déjà "pris» par un autre doctorant, Émilien Constant.

\section{Comprendre la République au village}

C'est dans l'histoire de la Provence qu'il puise dès lors une partie de son inspiration. Deux sections de la bibliothèque sont donc destinées à l'histoire de l'espace méridional français : «Héritage régional : le Var, Marseille, Avignon» et «Héritage régional : Provence, Midi». Ces héritages régionaux témoignent des lieux de naissance, de résidence et de travail de Maurice Agulhon et sont devenus les espaces de prédilection de ses recherches. Relevons la place des ouvrages concernant les révolutions du XIX ${ }^{\mathrm{e}}$ siècle à Marseille et Toulon : on compte onze ouvrages pour Marseille et vingt-et-un pour Toulon. Il ne s'agit pas ici de revenir sur le cheminement intellectuel qui a conduit Maurice Agulhon de questions très labroussiennes sur l'histoire économique aux recherches très anthropologiques sur la sociabilité : il importe ici seulement de souligner que sa bibliothèque en témoigne. Y figure notamment le livre de Lucienne Roubin, Chambrette des Provençaux ${ }^{11}$, qui a joué un rôle majeur pour le mettre sur la voie de la compréhension du passage du Midi blanc au Midi rouge ${ }^{12}$.

11. Lucienne Roubin, Chambrettes des Provençaux : une maison des hommes en Méditerranée septentrionale, Paris, Plon, 1970.

12. Maurice Agulhon, Histoire vagabonde 1. Ethnologie et politique dans la France contemporaine, Paris, Gallimard, 1988, p. 18. 
La sociabilité est un des concepts clefs de l'œuvre de Maurice Agulhon, concept inspiré d'ailleurs de Fernand Benoît dont les œuvres figurent sur les rayonnages de chacun des deux fonds Agulhon. Elle est au cour d'une autre section de la bibliothèque, où se trouvent nombre de travaux sur les pénitents, les confréries, les congrégations, la franc-maçonnerie et les sociétés secrètes. Maurice Agulhon a donc travaillé de manière pluridisciplinaire : on relève d'ailleurs dans sa bibliothèque la présence d'ouvrages d'anthropologie et d'ethnologie - avec en bonne place Van Gennep -, mais aussi de sociologie et de science politique. La sociabilité demeure toutefois le sujet qui y est le mieux représenté. En érigeant coutumes et traditions folkloriques de Provence au rang d'objets d'étude pour l'anthropologie historique, Maurice Agulhon a brouillé les frontières entre les centres d'intérêt des érudits locaux et des historiens. Il a redonné ses lettres de noblesse à l'histoire locale en démontrant que l'anthropologie historique avait beaucoup à y apprendre. Il est, ce faisant, devenu aussi l'un des meilleurs spécialistes de la Deuxième République, régime dans lequel il voit naître les principales forces politiques qui animent la vie politique actuelle. Comme attendu, une part importante de la bibliothèque lui est donc consacrée.

L'année 1848 a sa propre section dans la bibliothèque, additionnée de ce qui concerne le romantisme, et suivie d'une autre section qui va de la Révolution française à la Commune. Ces deux sections rassemblent des sources essentielles, de Chateaubriand ou Flora Tristan à Maxime Du Camp ou Pierre Dupont, en passant par la "parole ouvrière ${ }^{13}$. Elles comportent aussi une part importante de la production scientifique des historiens sur le sujet, d'Albert Charles et Régine Robin à Louis Hincker, Laurent Le Gall ou Sylvie Aprile, en passant par les productions du Centre aixois d'études et de recherches sur le XVIII ${ }^{e}$ siècle, le livre de Louis Girard sur Les libéraux français (1985), le Dictionnaire critique de la Révolution française de François Furet et Mona Ozouf (1988), l'œuvre de Michel Vovelle et celle de Daniel Roche, etc.

Ces sections de la bibliothèque mettent enfin à l'honneur les travaux de Maurice Agulhon et soulignent notamment qu'il a été le commissaire de l'exposition dédiée aux cent-cinquante ans de la révolution de 1848, organisée à l'Assemblée nationale. Deux catalogues ont vu le jour à la suite de cette exposition : Les révolutions de 1848 et Le printemps des peuples. Dans cette section, des travaux collectifs et des ouvrages individuels sont dans la continuité des recherches agulhoniennes et rendent hommage à celles-ci : le catalogue de l'exposition Armand Barbès et la révolution de 1848 ou 1848, la révolution oubliée de Maurizio Gribaudi et Michèle Riot-Sarcey. L'influence du travail de Maurice Agulhon est également manifeste dans la Revue d'histoire $d u X I X^{e}$ siècle, dont chaque nouveau numéro vient aujourd'hui encore 
enrichir la bibliothèque. En conjuguant histoire sociale, histoire politique et anthropologie, Maurice Agulhon a renouvelé l'approche locale en histoire et a donné une place essentielle aux acteurs du quotidien dont les luttes villageoises ont des répercussions nationales. Mais celui-ci, dès la fin des années 1970, se lance dans une entreprise historique novatrice : l'association de l'histoire politique et de l'histoire symbolique.

\section{De Marianne à de Gaulle}

Maurice Agulhon a inventé une nouvelle approche pour étudier l'histoire républicaine, en associant histoire de l'art et histoire politique. L'imagerie républicaine permet en effet de porter un regard neuf sur l'histoire politique des $\mathrm{XIX}^{\mathrm{e}}$ et $\mathrm{XX}^{\mathrm{e}}$ siècles. Les messages et symboles politiques ne sont pas le monopole des documents écrits. Ils sont portés aussi par des images, objets et représentations. Une partie du patrimoine du XIX et du XXe siècles garde encore une dimension encomiastique. Elle ne fait plus l'éloge du prince, comme sous l'Ancien Régime, mais celui du nouveau régime politique : la République. La section "théories de l'imagerie» comprend un grand nombre d'ouvrages consacrés aux monuments rendant hommage aux soldats morts pour la patrie : Les Monuments aux morts : patrimoine et mémoire de la Grande guerre d'Annette Becker; Mémoire figée, mémoire vivante: les monuments aux morts de Jacques Bouillon et Michel Petzold; Mémoires de pierre: les monuments aux morts de la première guerre mondiale dans le Pas-de-Calais; Les monuments aux morts de la guerre 1914-1918 dans le Vaucluse; La France et ses morts : les monuments commémoratifs dans la Loire de Monique Luirard. Maurice Agulhon a préfacé l'ouvrage de Michel Turc sur un monument aux morts en Provence qui s'intitule Un chef-d'œuvre d'art public. Le monument aux morts de Saint-Rémy-de-Provence.

L'étude de l'iconographie du XIX siècle a amené l'auteur de La République au village à se tourner vers l'histoire de la photographie, qui devient très vite un art et un support de symboles politiques. La section des «théories de l'imagerie» compte donc plusieurs ouvrages sur le sujet : Regard d'un Parisien sur la Commune; La photographie, la peinture, la guerre: correspondance inédite de Crimée, 1855-1856 de Jean-Charles Langlois; trois ouvrages d'André Rouillé. Mais cette section est composée majoritairement d'essais et de traités sur les liens entre imagerie, symbole, politique et travail de l'historien. On peut citer une publication du Centre d'histoire culturelle des sociétés contemporaines sur La place des images en histoire?, mais aussi Iconographie et histoire des mentalités, Images et politique en France au XXe siècle, de Christian Delporte, Les objets racontent l'histoire, de Jean Garrigues et Marie-Hélène Baylac, L'historien et les images, de Francis Haskell, les actes du colloque Histoire, images, imaginaires: fin $X V^{k}$ siècle-début $X X^{e}$ siècle, dirigés par Michèle Ménard et Annie Duprat. 
Le travail que Maurice Agulhon a consacré à l'imagerie républicaine est focalisé sur la figure de Marianne. Il montre qu'un sentiment proche de l'affection s'est manifesté entre les citoyens français et une statue personnifiée. Toute une section de la bibliothèque, "Marianne et Bardot» est dédiée aux allégories et métamorphoses de Marianne au XIXe et XXe siècles. Des ouvrages évoquent l'histoire de la statue de la Liberté dans la mesure où il existe un lien de famille entre la «déesse» française et la «déesse» américaine : une biographie de Bartholdi, Bartholdi and the statue of Liberty, La Statue de la Liberté, ou encore Liberty : the French-American Statue in Art and History. La section sur Marianne est surtout révélatrice de la multiplicité des supports sur lesquels l'allégorie de la Liberté a été représentée : ses ouvrages portent sur des tableaux, dont le plus connu de tous est celui de Delacroix La Liberté guidant le peuple, des statues, des bustes dans les mairies, des monnaies, des médailles, des billets, des timbres-poste, des fêtes, sans négliger les prénoms des nouveaux-nés. Les diverses manières de figurer la République sont au cœur de la documentation constituant cette section : les travaux de Pierre Bonte et de Jean Adhémar, avec qui Maurice Agulhon a fait équipe, le catalogue de l'exposition à l'Assemblée nationale Allégories de la République; deux catalogues d'exposition Quand Paris dansait avec Marianne: 1879-1889 et Un siècle de passions franco-allemandes: Marianne et Germania, 1789-1889; Les fées de la République de Jean-Michel Renault; le catalogue du Musée de la Monnaie La République fête son bicentenaire à la Monnaie. Les représentations de Marianne sont diverses et elles couvrent tout le territoire français. Des ouvrages en témoignent : Marianne dans les mairies du Rhône; Les Mariannes de la République en Franche-Comté; Les Mariannes de l'Hérault et leur place dans l'histoire; Marianne en pays saint-pourcinois. Visages et villages de la République.

Maurice Agulhon a été particulièrement soucieux d'évoquer dans ses travaux l'incarnation de la figure de Marianne dans un corps physique, féminin. Cette incarnation a été immortalisée entre autres par l'actrice française Brigitte Bardot. Les livres sur cette icône féminine des années 1960 ont une place dans cette section : Initiales B.B. : mémoires; Le Carré de Pluton et Un cri dans le silence; figure aussi une biographie de Brigitte Bardot, Brigitte Bardot: un mythe français. Si l'on considère le fonds Maurice Agulhon comme un patrimoine écrit et matériel, il faut alors ajouter à la section sur Marianne toutes les images et bustes que l'historien a conservés dans sa bibliothèque et dans son bureau : $s^{\prime} y$ trouve notamment une peinture représentant une figure féminine tenant le drapeau tricolore. Elle est debout sur un globe terrestre et les représentants des nations étrangères lèvent les yeux vers le ciel pour l'admirer.

Dans les années 1990-2000, Maurice Agulhon a réussi à conjuguer l'histoire politique, l'histoire de l'art et l'histoire symbolique. Cependant, il crée une rupture dans son œuvre puisque son objet d'étude, dans les années 2000, 
n'est plus une allégorie républicaine mais un homme politique, Charles de Gaulle. Son optique vis-à-vis de l'homme du 18 juin est novatrice : il ne réalise pas une simple biographie de Charles de Gaulle, mais une étude sur les mythes et les imaginaires politiques qui entourent ce dernier. À travers la personne de De Gaulle, il dévoile la construction de la figure du grand homme. Il n'hésite pas à comparer la vie des grands hommes : il établit, notamment, un rapprochement entre la figure de Léon Gambetta et celle de Charles de Gaulle.

La section "de Gaulle» rassemble tous les textes, documents, livres sur le général que Maurice Agulhon a utilisés. On a, tout d'abord, les textes écrits par de Gaulle : les Mémoires; les Lettres, notes et carnets; les Articles et écrits; les Discours et messages. Puis, les ouvrages édités par des institutions qui commémorent le souvenir du "Grand Charles» comme l'Institut Charles de Gaulle ou la Fondation Charles de Gaulle. Enfin, de nombreuses biographies, plus ou moins partisanes, telle la biographie en trois volumes de Jean Lacouture. Notons aussi la présence des entretiens avec Michel Debré ou les souvenirs d'Alain Peyrefitte. Grand donateur d'ouvrages à Maurice Agulhon, s'il est un adversaire politique, il est aussi un "associé» dans la réflexion sur de Gaulle ${ }^{14}$.

\section{Les cheminements divers}

Maurice Agulhon ne doit pas être perçu seulement comme un historien de la République et il ne se reconnaît pas lui-même uniquement comme tel ${ }^{15}$. Du reste, pour appréhender l'histoire républicaine, il s'est référé, on l'a vu, à la sociabilité mais aussi à la franc-maçonnerie, à l'histoire des intellectuels, à la laïcité, autant de thèmes qui renvoient aussi à l'anthropologie historique. Partant de ces champs, Maurice Agulhon a donc abordé des sujets autres que ceux qui font sa principale identité et qui sont au cœur de renouvellements de l'historiographie française. La section "XIXe-XXe siècle par thèmes : femmes, corps, hommes, animaux» en est l'illustration. Il s'inscrit ainsi dans l'histoire des femmes, puisque ses études sur Marianne l'ont amené à analyser l'iconographie féminine. Un de ses articles est un exemple de cette démarche: "Un usage de la femme au XIX siècle : l'allégorie de la République» ${ }^{16}$. Il a également écrit un autre article sur l'imagerie féminine qui prend en compte l'héritage révolutionnaire ${ }^{17}$. N'oublions pas qu'il appartient à la génération de Madeleine Rebérioux et Michelle Perrot, pionnières de l'histoire des femmes

14. Selon les termes employés par Maurice Agulhon lui-même.

15. Maurice Agulhon, Histoire et politique à gauche, Paris, Perrin, 2005, p. 21.

16. Maurice Agulhon, "Un usage de la femme au XIX“ siècle : l’allégorie de la République», Romantisme, $\mathrm{n}^{\circ} 13-14,1976$, p. 143-152.

17. Maurice Agulhon, "L'image féminine de la Révolution au XIXe siècle» in Marie-France Brive [dir.], Les Femmes et la Révolution française, Toulouse, Presses universitaires du Mirail, 1991, tome 3, p. 95-102. 
en France. Des disciples de Maurice Agulhon comme Anne-Marie Sohn ont d'ailleurs inscrit une partie de leur œuvre dans les cadres de la gender history.

L'historien de la révolution de 1848 s'est intéressé aussi à l'histoire des animaux. La revue Sociétés et représentations a même réalisé un entretien avec Maurice Agulhon, en 2008, autour de la question des rapports entre ethnologie, histoire et animalitée ${ }^{18}$. Il a publié deux articles s'intéressant au traitement des animaux et aux violences faites à ces derniers en 1981 et en $1996^{19}$. Ses analyses ont permis de confirmer que les animaux en France sont sources de symboles et de valeurs politiques. Il a également établi un parallèle entre les violences faites aux animaux et les violences faites aux êtres humains. Pour construire son argumentation, il s'appuie sur des livres traitant de l'histoire de la corrida, de la peur des hommes vis-à-vis des loups ou des échanges entre les animaux et les hommes ${ }^{20}$.

\section{UN FONDS DE TRAVAIL ET D'ÉCHANGES, REFLET DES RÉSEAUX D'UN HISTORIEN}

\section{Les maîtres}

La lecture de l'inventaire de la bibliothèque de Maurice Agulhon révèle non seulement l'environnement intellectuel de sa formation et de ses recherches, mais aussi l'espace de relations dans le cadre duquel il a évolué. On peut en effet s'attendre à trouver dans cette bibliothèque la trace de ses mentors, de Joseph Hours à Ernest Labrousse en passant par Louis Girard, Pierre Vilar ou encore Pierre Guiral. Mais celui qui lui a fait découvrir vraiment l'histoire, au lycée du Parc, à Lyon, et qui l'a conduit à la choisir pour discipline, Joseph Hours, a peu écrit. Il est néanmoins présent dans sa bibliothèque par son travail sur Le mouvement ouvrier français et par sa Petite histoire du mouvement ouvrier, sélection fort révélatrice. Ernest Labrousse n'est présent que par le volume d'hommages qui a été publié en son honneur. Louis Girard n’apparaît qu'avec son volume de la Nouvelle histoire de Paris. L'œuvre de Henri-Irénée Marrou figure avec deux éditions de La Connaissance historique, celle de 1955 et celle de 1975. Celle de Marcel Reinhardt est représentée par deux ouvrages, celui qui porte sur l'enseignement de

18. Maurice Agulhon, "Un historien et l'ethnologie», Sociétés \& Représentations, n 27, 2009/1, p. 239-247.

19. Maurice Agulhon, «Le sang des bêtes : le problème de la protection des animaux en France au $\mathrm{XIX}^{\mathrm{e}}$ siècle», Romantisme, $\mathrm{n}^{\circ}$ 31, 1981, p. 81-109; «Une histoire oubliée la protection des animaux comme pédagogie de la non-violence au XIX siècle", Bulletin annuaire de la société d'histoire et d'archéologie du Vieux Villeneuve, 1996.

20. Eric Baratay et Elisabeth Hardoin-Fugier, La corrida, Paris, Presses universitaires de France, 1995; Auguste Lafront, Histoire de la corrida en France, du Second Empire à nos jours, Paris, Julliard, 1977; Daniel Bernard, L'homme et le loup, Paris, Berger-Levrault, 1981; Florence Burgat, La protection de l'animal, Paris, Presses universitaires de France, 1997. 
l'histoire, publié en 1957, et celui qui est consacré à la population française aux XVIII ${ }^{\mathrm{e}}$ et XIX ${ }^{\mathrm{e}}$ siècles, paru en 1973.

Pierre Guiral, qui a accueilli Maurice Agulhon comme collègue à Aix-enProvence, est en revanche davantage présent, notamment par ses volumes sur la vie quotidienne des domestiques, des députés, des professeurs, par son travail sur Prévost-Paradol, par sa biographie de Thiers et par son Clemenceau en son temps. Comme Ernest Labrousse, il apparait aussi par le volume d'hommages qui ont été réunis par ses disciples. Contrairement aux ouvrages des auteurs précédents, ceux-ci portent des dédicaces sobres mais qui témoignent $\mathrm{du}$ lien fidèlement maintenu entre deux hommes nourrissant un très sincère respect mutuel. Les maitres deviennent donc pour certains des proches.

\section{Les pairs et les amis}

Les pairs qui figurent dans la bibliothèque de Maurice Agulhon sont d'abord ceux avec qui il a tissé des liens lorsqu'il était en khâgne à Lyon puis rue d'Ulm. Se trouvent donc en bonne place sur les rayons de Villeneuve les ouvrages du philosophe Lucien Sève, rencontré à Lyon, et qui a été en définitive l'ami qui a le plus compté dans sa jeunesse. La mention de «fidèle amitié» orne chacun des livres envoyés par Lucien Sève. À l'École normale supérieure, il se lie particulièrement avec Bernard Guenée, qui lui témoigne lui aussi, avec chaque envoi de ses ouvrages, sa "fidèle amitié». Il est alors également proche de François Bédarida, qui lui adresse lui aussi ses œuvres avec amitié et fidélité. Il côtoie aussi Jacques Le Goff et Alain Peyrefitte, de la promotion antérieure à la sienne, mais qui ne lui en témoignent pas moins eux aussi leur amitié par leurs envois. Des liens se nouent de même avec les autres élèves d'Ernest Labrousse, notamment Michelle Perrot qui envoie ses livres «avec [s] on amitié", qui devient fidèle.

Viennent ensuite les amis en politique, au premier rang desquels il faut mentionner Annie Kriegel, qui n'hésite pas à lui adresser ses ouvrages en parlant d'affectueux compagnonnage ou de fraternel hommage et de fidèle amitié. Cette amitié s'est nouée également avec le frère d'Annie Becker, JeanJacques. L'amitié entre les deux hommes est restée vive jusqu’à nos jours, même si elle s'exprime avec retenue dans les dédicaces qui soulignent une "si longue amitié». Celle-ci a du reste été transmise à la nouvelle génération puisque Annette Becker figure elle aussi en bonne place dans la bibliothèque : ses ouvrages évoquent l'affection et l'amitié qui l'unissent à l'ami de son père. En outre, les engagements politiques ont conduit Maurice Agulhon à côtoyer Robert Bonnaud, membre du parti communiste qui engagea son ami y compris sur la voie des porteurs de valises ${ }^{21}$. En dépit du fait que ce cheminement commun n'a pas duré longtemps, Robert Bonnaud a conservé ces liens avec Maurice Agulhon, et lui a envoyé ses livres en gage de son ami- 
tié fidèle. Madeleine Rebérioux, historienne elle aussi, a de même rencontré Maurice Agulhon autour de préoccupations politiques communes, et fait allusion dans ses envois à leur "amitié jaurésienne». Des liens ont été tissés avec les Aubrac, et Lucie envoie ses livres «amicalement», Charles Tillon le fait aussi avec ses «sentiments de fraternelle amitié», Robert Brécy invoque leur amitié, Michel Verret ${ }^{22}$ souligne plus fortement encore leur vieille et fidèle amitié.

La bibliothèque témoigne également des relations et amitiés qui se sont nouées à Aix-en-Provence, en premier lieu avec Michel Vovelle, ami aujourd'hui encore régulièrement présent, et dont les envois disent la fidèle et fraternelle amitié qui unit les deux hommes, lesquels sont ensemble, au fil de leurs recherches, passés de la cave au grenier. Les années aixoises ont valu pareillement à Maurice Agulhon l'amitié d'Yvonne Knibiehler, de Louis Stouff ou de Bernard Cousin, mais aussi, bien évidemment, de Georges Duby, qui lui ouvre ensuite bien des portes parisiennes. Là encore, les dédicaces emploient sans réserve le mot d' "amitié». Viennent ensuite les années parisiennes, celles de l'université Paris I tout d'abord, où se font les rencontres avec Louis Girard, Claude Fohlen ou Jean-Marie Mayeur. L'ambiance n'est plus à la proximité aixoise et les livres témoignent alors de relations amicales, qui ne vont pas jusqu'à l'amitié. Quant aux pairs du Collège de France, ils sont nombreux à se retrouver dans la bibliothèque de Villeneuve-lez-Avignon : André Miquel fait ses envois "en très fidèle hommage ", Marc Fumaroli le fait "très amicalement ", Yves Coppens lui dit un hommage très sincère ou un souvenir amical, Emmanuel Le Roy Ladurie s'adresse "au vieux camarade de combat et d'espérance», en le faisant selon les cas "très amicalement" ou "très affectueusement ", Pierre Bourdieu, Jean-Pierre Vernant comme Daniel Roche attestent pour leur part de leur amitié. Il y a aussi les pairs en histoire de la Deuxième République, tels Philippe Vigier ou Pierre Lévêque. Institutionnellement plus lointains, Robert Mandrou dit pourtant son amitié et Jean Nicolas sa «fraternité rébellionnaire» et sa fidèle amitié.

La bibliothèque de Maurice Agulhon témoigne enfin des liens noués avec des collègues étrangers comme Charles Tilly, qui l'assure de ses hommages amicaux, Théodore Zeldin, qui proclame sa vive sympathie, tandis que Lawrence Stone dit son admiration et son affection, Eugen Weber son amitié et son admiration. Arno Mayer quant à lui signe simplement et clairement «ton ami ». La collection témoigne aussi des échanges avec les ethnologues comme Marie-Hélène Froeschlé-Chopard, Lucienne Roubin, Martine Segalen ou encore Jean Cuisenier, conservateur au Musée national des Arts et Traditions populaires de Paris. Elle rend compte aussi des amitiés éditoriales avec

22. Sur le parcours de Michel Verret au sein du communisme, voir «Histoire d'une fidélité. Biographie de Michel Verret par lui-même», Politix, 1991, n 3, p. 43-49. 
François Furet, aux côtés de qui Maurice Agulhon participe entre autres aux discussions suscitées par le bicentenaire de la Révolution, ou Pierre Nora, qui a notamment été à l'origine de l'essai d'ego histoire de Maurice Agulhon. Elle fait enfin apparaitre le respect des pairs plus jeunes comme Alain Corbin, Yves Lequin ou Ronald Hubscher.

\section{Les disciples et les admirateurs}

Une part importante de la bibliothèque est constituée des ouvrages envoyés par les disciples et les admirateurs de Maurice Agulhon et l'analyse de leur corpus nécessiterait bien plus que les quelques lignes qu'il est ici possible d'y consacrer. Plusieurs générations se succèdent parmi les disciples, y compris parmi les docteurs de Maurice Agulhon. Mais ceux d'entre eux qui n'ont pas poursuivi dans la carrière d'historien sont absents de la bibliothèque. Jacqueline Lalouette est la première disciple - par ordre chronologique de soutenance des thèses - dont les ouvrages figurent sur les rayonnages de Villeneuve. Elle envoie ses livres accompagnés de l'expression de son affection et de sa gratitude. Christophe Prochasson est bien moins présent qu'elle dans la bibliothèque et signe avec un simple «bien amicalement». Laurent Douzou exprime sa haute estime et sa reconnaissance puis se permet de témoigner de sa fidèle amitié, ce que fait également Marcel Vigreux. Christophe Charle, que Maurice Agulhon souhaitait voir lui succéder au Collège de France, dit sa fidèle amitié mais aussi son admiration. Jocelyne George le remercie pour ses encouragements et le salue respectueusement et fidèlement. Jacques Girault signe avec son amitié, Anne-Marie Sohn, qui n'ose pas initialement plus que des hommages respectueux, revendique chaleureusement, en une dédicace plus récente, son statut d' «élève». Jean-François Chanet affiche lui aussi sa position d' "élève», et dit son respect, sa gratitude et son amitié. Notons enfin la présence des ouvrages de Jacques Rougerie, l'un des rares à afficher le tutoiement dans ses dédicaces, et qui, s'il n'est pas un docteur de Maurice Agulhon, partage avec lui une grande complicité d'historien des révolutions du XIX ${ }^{\mathrm{e}}$ siècle et de la République.

Le fonds recueille également tous les ouvrages envoyés en signe d'admiration par un grand nombre d'historiens du contemporain, parmi lesquels se distinguent bien évidemment les historiens du XIX ${ }^{e}$ siècle, de JeanClaude Caron à Sylvie Aprile et Francis Démier, de Raymond Huard à Louis Hincker, de Yannick Marec à Laurent Le Gall, de Jean-Luc Mayaud à Jordi Canal. Même les tenants du courant dominé par Alain Corbin sont présents. $\mathrm{Du}$ reste, figurent aussi systématiquement dans la bibliothèque les exemplaires dédicacés par les auteurs de la collection «Historique», chez Aubier, co-dirigée par Maurice Agulhon. Alain Corbin lui-même y a publié et ne manque pas d'exprimer ses hommages respectueux puis amicaux. Les auteurs de la collection disent tous leur dette à l'égard de Maurice Agulhon qui les a édités. La bibliothèque de Villeneuve prouve aussi que les historiens du local 
reconnaissent bien volontiers leur dette à son égard, tel Félix Laffé ou Thérèse Moyne. Elle révèle que même les amateurs osent lui adresser leur production, tel François Sarda. Elle souligne enfin le rayonnement de Maurice Agulhon sur l'ensemble des contemporanéistes, mais aussi des dix-huitiémistes. En parcourant ses rayonnages, on finit par rencontrer tous les historiens, jeunes et moins jeunes, qui publient sur ces périodes, tels Arlette Farge, Robert Muchembled, Michel Figeac, Annie Duprat, Francis Démier, Odile Rudelle, André Encrevé, Rémi Dalisson, Philippe Darriulat, Simone Delattre, Jean Garrigues, Bruno Dumons, Pierre Birnbaum, Christian Delporte, Serge Berstein, Danielle Tartakowsky, John Keegan, etc... Les hommages viennent de toutes parts, y compris d'Afrique (Abdou Touré), de Corée (Chun Soo Yun) ou d'Amérique (Olivier Zunz), pour dire tous que Maurice Agulhon est à jamais au cour du paysage historiographique de la France, des années 1960 aux années 2010. Cela éclaire aussi l'intérêt que lui manifestent des hommes politiques tels que Lionel Jospin, Anicet Le Pors, Roger Martelli, Jean Poperen, Gérard Streiff, ou Philippe Séguin - mais celui-ci a été, il est vrai, l'élève de Maurice Agulhon à Aix-en-Provence. Même les écrivains lui font hommage de leurs livres, tel Gonzague Saint-Bris lorsqu'il évoque les romantiques.

La présentation qui précède n'est qu'une première approche qui est loin d'épuiser tout ce que peut apprendre la bibliothèque de Maurice Agulhon sur la vie et l'œuvre de celui-ci ${ }^{23}$. Elle a avant tout pour but de faire connaitre l'existence de ce fonds d'une part, de rendre hommage, d'autre part, à l'œuvre immense de son propriétaire. Parce que sa bibliothèque démontre l'ampleur des champs dans lesquels s'inscrit son travail ainsi que la force de son rayonnement, elle mérite d'être connue de tous les historiens et amateurs du XIX ${ }^{e}$ siècle. Du reste, ce fonds prouve également à quel point Maurice Agulhon ne doit pas être identifié uniquement comme spécialiste du $\mathrm{XIX}^{\mathrm{e}}$ siècle : sa bibliothèque rappelle qu'il est l'un des historiens qui a le plus apporté aux réflexions sur les temps contemporains, de la Révolution française à nos jours. Les envois et dédicaces innombrables de tous les auteurs qui ont contribué à enrichir nos connaissances sur le sujet en témoignent.

Reste que la constitution de la bibliothèque de Villeneuve-lez-Avignon en véritable patrimoine est un problème non résolu. Pour l'heure, elle ne peut être visitée que de façon virtuelle, et Maurice Agulhon n'a pas encore pu résoudre la question de ce qu'elle deviendra une fois que ses descendants en seront les détenteurs. Il demeure très préoccupé de la façon dont elle

23. Précisons toutefois que Maurice Agulhon n'est nullement coutumier des annotations en marge des ouvrages qu'il lit. Il y place des quarts de feuille que l'on ne retrouve que dans de rares ouvrages. 
pourrait être accessible aux chercheurs à partir du moment où sa maison ne sera plus habitée que ponctuellement. Quant à une donation à l'université d'Avignon qui l'a inventoriée et filmée, elle n'est nullement à l'ordre du jour.

Jonathan Barbier est doctorant en histoire contemporaine, Natalie Petiteau est professeur d'histoire contemporaine à l'université d'Avignon, Centre Norbert Elias 